\title{
Second-order duality for nondifferentiable minimax fractional programming problems with generalized convexity
}

Meraj Ali Khan*

"Correspondence:

meraj79@gmail.com

Department of Mathematics,

University of Tabuk, Tabuk, Kingdom

of Saudi Arabia

\begin{abstract}
In the present paper, we are concerned with second-order duality for nondifferentiable minimax fractional programming under the second-order generalized convexity type assumptions. The weak, strong and converse duality theorems are proved. Results obtained in this paper extend some previously known results on nondifferentiable minimax fractional programming in the literature.

MSC: $90 \mathrm{C} 32 ; 49 \mathrm{~K} 35 ; 49 \mathrm{~N} 15$

Keywords: minimax programming; fractional programming; duality; generalized convexity
\end{abstract}

\section{Introduction}

It is well known that the minimax fractional programming has wide applications. These types of problems arise in the design of electronic circuits; moreover, minimax fractional programming problems appear in formulation of discrete and continuous rational approximation problems with respect to the Chebyshev norm [1], continuous rational games [2], multiobjective programming [3] and engineering design as well as some portfolio solution problems discussed by Bajaona-Xandari and Martinez-Legaz [4].

In the last few years, much attention has been paid to optimality conditions and duality theorems for the minimax fractional programming problems. For the case of convex differentiable minimax fractional programming, Yadav and Mukherjee [5] formulated two dual models for the primal problem and derived a duality theorem for convex differentiable minimax fractional programming. A step forward was taken by Chandra and Kumar [6] who improved the dual formulation of Yadav and Mukherjee. They provided two modified dual problems for minimax fractional programming and proved duality results. Liu and Wu [7, 8] and Ahmad [9] obtained sufficient optimality conditions and duality for minimax fractional programming under generalized convex type assumptions.

Mangasarian [10] introduced the notion of second-order duality for nonlinear programs and obtained second-order duality results under certain inequalities. Mond [11] modified the second-order duality results assuming rather simple inequalities. In this continuation, Bector and Chandra [12] formulated a second-order dual for a fractional programming problem and obtained usual duality results under the assumptions [10] by naming these as convex/concave functions. Recently, Ahmad [13] has formulated two types of secondorder dualities for minimax fractional programming problems and derived weak, strong

o2013 Khan; licensee Springer. This is an Open Access article distributed under the terms of the Creative Commons Attribution License (http://creativecommons.org/licenses/by/2.0), which permits unrestricted use, distribution, and reproduction in any medium, provided the original work is properly cited. 
and strict converse duality theorems under generalized convexity type assumptions. $\mathrm{He}$ raised a question as to whether the second-order duality results developed in [13] hold for nondifferentiable minimax fractional programming problems. In the present paper, a positive answer is given to the question of Ahmad [13] and a second-order duality for nondifferentiable minimax fractional programming is formulated. The weak, strong and strict converse duality theorems are proved for these programs under the second-order generalized convexity type assumptions.

We consider the following nondifferentiable minimax fractional programming problem:

$$
\begin{aligned}
& \text { Minimize } \quad \psi(x)=\sup _{y \in Y} \frac{f(x, y)+\left(x^{T} C x\right)^{\frac{1}{2}}}{g(x, y)-\left(x^{T} D x\right)^{\frac{1}{2}}} \\
& \text { subject to } \quad h(x) \leq 0, \quad x \in R^{n},
\end{aligned}
$$

where $Y$ is a compact subset of $R^{l}, f(\cdot, \cdot), g(\cdot, \cdot): R^{n} \times R^{l} \rightarrow R, h(\cdot, \cdot): R^{n} \rightarrow R^{m}$ are $C^{1}$ functions. $C$ and $D$ are $n \times n$ positive semidefinite symmetric matrices. Throughout this paper, we assume that $g(x, y)+\left(x^{T} D x\right)^{\frac{1}{2}}>0$ and $f(x, y)+\left(x^{T} C x\right)^{\frac{1}{2}} \geq 0$ for all $(x, y) \in R^{n} \times R^{l}$.

\section{Notations and preliminaries}

Let $S=\left\{x \in R^{n}: h(x) \leq 0\right\}$ denote the set of all feasible solutions of (NFP), a point $x \in S$ is called the feasible point of (NFP). For each $(x, y) \in R^{n} \times R^{l}$, we define

$$
J(x)=\left\{j \in M: h_{j}(x)=0\right\},
$$

where $M=\{1,2, \ldots, m\}$,

$$
\begin{aligned}
Y(x)= & \left\{y \in Y: \frac{f(x, y)+\left(x^{T} C x\right)^{\frac{1}{2}}}{g(x, y)-\left(x^{T} D x\right)^{\frac{1}{2}}}=\sup _{z \in Y} \frac{f(x, z)+\left(x^{T} C x\right)^{\frac{1}{2}}}{g(x, z)-\left(x^{T} D x\right)^{\frac{1}{2}}}\right\}, \\
K(x)= & \left\{(s, t, \bar{y}) \in N \times R_{+}^{s} \times R^{l s}: 1 \leq s \leq n+1, t=\left(t_{1}, t_{2}, \ldots t_{s}\right) \in R_{+}^{s}\right. \\
& \text { with } \left.\sum_{i=1}^{s} t_{i}=1, \bar{y}=\left(\bar{y}_{1}, \ldots, \bar{y}_{s}\right) \text { where } \bar{y}_{i} \in Y(x), i=1,2, \ldots, s\right\} .
\end{aligned}
$$

Since $f$ and $g$ are $C^{1}$ functions and $Y$ is compact in $R^{l}$, it follows that for each $x^{*} \in S$, $Y\left(x^{*}\right) \neq \phi$, and for any $\bar{y}_{i} \in Y\left(x^{*}\right)$, we have a positive constant

$$
K_{0}=\psi\left(x^{*}, \bar{y}_{i}\right)=\frac{f\left(x^{*}, \bar{y}_{i}\right)+\left(x^{* T} C x^{*}\right)^{\frac{1}{2}}}{g\left(x^{*}, \bar{y}_{i}\right)-\left(x^{* T} D x^{*}\right)^{\frac{1}{2}}} .
$$

\section{Generalized Schwarz inequality}

Let $A$ be a positive semidefinite matrix of order $n$. Then, for all $x, w \in R^{n}$,

$$
x^{T} A w \leq\left(x^{T} A x\right)^{1 / 2}\left(w^{T} A w\right)^{1 / 2} .
$$

Equality holds if, for some $\lambda \geq 0$,

$$
A x=\lambda A w .
$$


Evidently, if $\left(w^{T} A w\right)^{1 / 2} \leq 1$, we have

$$
x^{T} A w \leq\left(x^{T} A x\right)^{1 / 2} .
$$

Definition 2.1 A function $\mathcal{F}: X \times X \times R^{n} \rightarrow R$, where $X \subseteq R^{n}$ is said to be sublinear in its third argument if $\forall x, \bar{x} \in X$,

(i) $\mathcal{F}\left(x, \bar{x} ; a_{1}+a_{2}\right) \leq \mathcal{F}\left(x, \bar{x} ; a_{1}\right)+\mathcal{F}\left(x, \bar{x} ; a_{2}\right), \forall a_{1}, a_{2} \in R^{n}$,

(ii) $\mathcal{F}(x, \bar{x} ; \alpha a)=\alpha \mathcal{F}(x, \bar{x} ; a), \forall \alpha \in R_{+}, a \in R^{n}$.

Definition 2.2 A point $\bar{x} \in S$ is said to be an optimal solution of (NFP) if $\psi(x) \geq \psi(\bar{x})$ for each $x \in S$.

In the case where the functions $f, g$ and $h$ in problem (NFP) are continuously differentiable with respect to $x \in R^{n}$, Lai et al. [14] proved the following first-order necessary conditions for optimality of (NFP), which will be required to prove the strong duality theorem.

Theorem 1 (Necessary condition) Let $x^{*}$ be a solution (local or global) of (NFP) satisfying $x^{* T} C x^{*}>0, x^{* T} D x^{*}>0$, and let $\nabla h_{j}\left(x^{*}\right), j \in J\left(x^{*}\right)$ be linearly independent. Then there exist $\left(s^{*}, t^{*}, \bar{y}^{*}\right) \in K\left(x^{*}\right), k_{0} \in R_{+}, w, v \in R^{n}$ and $\mu^{*} \in R_{+}^{m}$ such that

$$
\begin{aligned}
& \sum_{i=1}^{s^{*}} t_{i}^{*}\left\{\nabla f\left(x^{*}, \bar{y}_{i}^{*}\right)+C w-k_{0}\left(\nabla g\left(x^{*}, \bar{y}_{i}^{*}\right)-D v\right)\right\}+\nabla \sum_{j=1}^{m} \mu_{j}^{*} h_{j}\left(x^{*}\right)=0, \\
& f\left(x^{*}, \bar{y}_{i}^{*}\right)+\left(x^{* T} C x^{*}\right)^{\frac{1}{2}}-k_{0}\left(g\left(x^{*}, \bar{y}_{i}^{*}\right)-\left(x^{* T} D x^{*}\right)^{\frac{1}{2}}\right)=0, \quad i=1,2, \ldots, s^{*}, \\
& \sum_{j=1}^{m} \mu_{j}^{*} h_{j}\left(x^{*}\right)=0, \\
& t_{i}^{*} \geq 0, \quad \sum_{i=1}^{s^{*}} t_{i}^{*}=1, \\
& w^{T} C w \leq 1, \quad v^{T} D v \leq 1, \quad\left(x^{* T} C x^{*}\right)^{1 / 2}=x^{* T} C w, \quad\left(x^{* T} D x^{*}\right)^{1 / 2}=x^{* T} D v .
\end{aligned}
$$

Throughout the paper, we assume that $\mathcal{F}$ is a sublinear functional. For $\beta=1,2, \ldots, r$, let $b, b_{0}, b_{\beta}: X \times X \rightarrow R_{+}, \phi, \phi_{0}, \phi_{\beta}: R \rightarrow R, \rho, \rho_{0}, \rho_{\beta}$ be real numbers, and let $\theta: R^{n} \times R^{n} \rightarrow R$.

\section{Parametric nondifferentiable fractional duality}

In this section, we consider the following dual to (NFP):

$$
\max _{(s, t, \bar{y}) \in K(z)} \sup _{(z, \mu, k, v, w, p) \in H_{1}(s, t, \bar{y})} k,
$$


where $H_{1}(s, t, \bar{y})$ denotes the set of all $(z, \mu, k, v, w, p) \in R^{n} \times R_{+}^{m} \times R_{+} \times R^{n} \times R^{n} \times R^{n}$ satisfying

$$
\begin{aligned}
& \sum_{i=1}^{s} t_{i}\left\{\nabla f\left(z, \bar{y}_{i}\right)+\nabla^{2} f\left(z, \bar{y}_{i}\right) p+C w-k\left(\nabla g\left(z, \bar{y}_{i}\right)+\nabla^{2} g\left(z, \bar{y}_{i}\right) p-D v\right)\right\} \\
& \quad+\sum_{j=1}^{m} \nabla \mu_{j} h_{j}(z)+\sum_{j=1}^{m} \nabla^{2} \mu_{j} h_{j}(z)=0, \\
& \sum_{i=1}^{s} t_{i}\left\{f\left(z, \bar{y}_{i}\right)-\frac{1}{2} p^{T} \nabla^{2} f\left(z, \bar{y}_{i}\right) p+z^{T} C w\right. \\
& \left.\quad-k\left(g\left(z, \bar{y}_{i}\right)-\frac{1}{2} p^{T} \nabla^{2} g\left(z, \bar{y}_{i}\right) p-z^{T} D v\right)\right\} \geq 0, \\
& \sum_{j=1}^{m} \mu_{j} h_{j}(z)-\sum_{j=1}^{m} \frac{1}{2} p^{T} \nabla^{2} \mu_{j} h_{j}(z) \geq 0, \\
& (s, t, \bar{y}) \in k(z), \quad \\
& w^{T} C w \leq 1, \quad v^{T} D v \leq 1, \quad\left(z^{T} C z\right)^{1 / 2}=z^{T} C w, \quad\left(z^{T} D z\right)^{1 / 2}=z^{T} C v .
\end{aligned}
$$

If, for a triplet $(s, t, \bar{y}) \in k(z)$, the set $H_{1}(s, t, \bar{y})=\phi$, then we define the supremum over it to be $-\infty$.

Theorem 2 (Weak duality) Let $x$ and $(z, \mu, k, v, w, s, t, \bar{y})$ be feasible solutions of (NFP) and (FD), respectively. Assume that there exist $\mathcal{F}, \theta, \phi, b$ and $\rho$ such that

$$
\begin{aligned}
& b(x, z) \phi\left[\sum_{i=1}^{s} t_{i}\left(f\left(x, \bar{y}_{i}\right)+x^{T} C w-k\left(g\left(x, \bar{y}_{i}\right)-x^{T} D v\right)\right)\right. \\
& -\sum_{i=1}^{s} t_{i}\left(f\left(z, \bar{y}_{i}\right)+z^{T} C w-k\left(g\left(z, \bar{y}_{i}\right)-z^{T} D v\right)\right) \\
& \left.-\sum_{j=1}^{m} \mu_{j} h_{j}(z)+\frac{1}{2} p^{T} \nabla^{2} \sum_{i=1}^{s} t_{i}\left(f\left(z, \bar{y}_{i}\right)-k g\left(z, \bar{y}_{i}\right)\right) p+\frac{1}{2} p^{T} \nabla^{2} \sum_{j=1}^{m} \mu_{j} h_{j}(z) p\right]<0 \\
& \Rightarrow \quad \mathcal{F}\left(x, z ; \sum_{i=1}^{s} t_{i}\left(\nabla f\left(z, \bar{y}_{i}\right)+\nabla^{2} f\left(z, \bar{y}_{i}\right) p+C w-k\left(\nabla g\left(z, \bar{y}_{i}\right)+\nabla^{2} g\left(z, \bar{y}_{i}\right) p-D v\right)\right)\right. \\
& \left.\quad+\sum_{j=1}^{m}\left(\nabla \mu_{j} h_{j}(z)+\nabla^{2} \mu_{j} h_{j}(z) p\right)\right) \\
& <-\rho\|\theta(x, z)\|^{2}
\end{aligned}
$$

Further assume that

$$
\begin{aligned}
& a<0 \quad \Rightarrow \quad \phi(a)<0, \\
& b(x, z)>0, \\
& \rho \geq 0 .
\end{aligned}
$$


Then

$$
\sup _{y \in Y} \frac{f(x, y)+\left(x^{T} C x\right)^{\frac{1}{2}}}{g(x, y)-\left(x^{T} D x\right)^{\frac{1}{2}}} \geq k .
$$

Proof Suppose to the contrary that

$$
\sup _{y \in Y} \frac{f(x, y)+\left(x^{T} C x\right)^{\frac{1}{2}}}{g(x, y)-\left(x^{T} D x\right)^{\frac{1}{2}}}<k .
$$

Then we have

$$
f\left(x, \bar{y}_{i}\right)+\left(x^{T} C x\right)^{\frac{1}{2}}-k\left(g\left(x, \bar{y}_{i}\right)-\left(x^{T} D x\right)^{\frac{1}{2}}\right)<0
$$

for all $\bar{y}_{i} \in Y$.

It follows from (5) that

$$
\sum_{i=1}^{s} t_{i}\left\{f\left(x, \bar{y}_{i}\right)+\left(x^{T} C x\right)^{\frac{1}{2}}-k\left(g\left(x, \bar{y}_{i}\right)-\left(x^{T} D x\right)^{\frac{1}{2}}\right)\right\} \leq 0 .
$$

By the Schwarz inequality, the above inequality yields

$$
\sum_{i=1}^{s} t_{i}\left(f\left(x, \bar{y}_{i}\right)+x^{T} C w-k\left(g\left(x, \bar{y}_{i}\right)-x^{T} D v\right)\right)<0 .
$$

Inequality (17) together with (8) gives

$$
\begin{aligned}
& \sum_{i=1}^{s} t_{i}\left(f\left(x, \bar{y}_{i}\right)+x^{T} C w-k\left(g\left(x, \bar{y}_{i}\right)-x^{T} D v\right)\right) \\
& \quad<0 \\
& \quad \leq \sum_{i=1}^{s} t_{i}\left(f\left(z, \bar{y}_{i}\right)-\frac{1}{2} p^{T} \nabla^{2} f\left(z, \bar{y}_{i}\right) p+z^{T} C w-k\left(g\left(z, \bar{y}_{i}\right)-\frac{1}{2} p^{T} \nabla^{2} g\left(z, \bar{y}_{i}\right) p-z^{T} D v\right)\right) .
\end{aligned}
$$

Now, using (9) in the above inequality, we get

$$
\begin{aligned}
& \sum_{i=1}^{s} t_{i}\left(f\left(x, \bar{y}_{i}\right)+x^{T} C w-k\left(g\left(x, \bar{y}_{i}\right)-x^{T} D v\right)\right) \\
& \quad-\sum_{i=1}^{s} t_{i}\left(f\left(z, \bar{y}_{i}\right)+z^{T} C w-k\left(g\left(z, \bar{y}_{i}\right)-z^{T} D v\right)\right) \\
& \quad-\sum_{j=1}^{m} \mu_{j} h_{j}(z)+\frac{1}{2} p^{T} \nabla^{2} \sum_{i=1}^{s} t_{i}\left(f\left(z, \bar{y}_{i}\right)-k g\left(z, \bar{y}_{i}\right)\right) p \\
& \quad+\frac{1}{2} p^{T} \nabla^{2} \sum_{j=1}^{m} \mu_{j} h_{j}(z) p<0 .
\end{aligned}
$$


Using (13) and (14), it follows from (18) that

$$
\begin{aligned}
& b(x, z) \phi\left[\sum_{i=1}^{s} t_{i}\left(f\left(x, \bar{y}_{i}\right)+x^{T} C w-k\left(g\left(x, \bar{y}_{i}\right)-x^{T} D v\right)\right)\right. \\
& \quad-\sum_{i=1}^{s} t_{i}\left(f\left(z, \bar{y}_{i}\right)+z^{T} C w-k\left(g\left(z, \bar{y}_{i}\right)-z^{T} D v\right)\right) \\
& \left.\quad-\sum_{j=1}^{m} \mu_{j} h_{j}(z)+\frac{1}{2} p^{T} \nabla^{2} \sum_{i=1}^{s} t_{i}\left(f\left(z, \bar{y}_{i}\right)-k g\left(z, \bar{y}_{i}\right)\right) p+\frac{1}{2} p^{T} \nabla^{2} \sum_{j=1}^{m} \mu_{j} h_{j}(z) p\right]<0,
\end{aligned}
$$

which along with (12) and (15) yields

$$
\begin{aligned}
& \mathcal{F}\left(x, z ; \sum_{i=1}^{s} t_{i}\left(\nabla f\left(z, \bar{y}_{i}\right)+\nabla^{2} f\left(z, \bar{y}_{i}\right) p+C w-k\left(\nabla g\left(z, \bar{y}_{i}\right)+\nabla^{2} g\left(z, \bar{y}_{i}\right) p-D v\right)\right)\right. \\
& \left.\quad+\sum_{j=1}^{m}\left(\nabla \mu_{j} h_{j}(z)+\nabla^{2} \mu_{j} h_{j}(z) p\right)\right)<0,
\end{aligned}
$$

which contradicts $(7)$, since $\mathcal{F}(x, z ; 0)=0$.

Theorem 3 (Strong duality) Let $x^{*}$ be an optimal solution of (NFP), and let $\nabla h_{j}\left(x^{*}\right), j \in$ $J\left(x^{*}\right)$ be linearly independent. Then there exist $\left(s^{*}, t^{*}, y^{*}\right) \in K\left(x^{*}\right)$ and $\left(x^{*}, \mu^{*}, k^{*}, v^{*}, w^{*}, p^{*}=\right.$ $0) \in H_{1}\left(s^{*}, t^{*}, \bar{y}^{*}\right)$ such that $\left(x^{*}, \mu^{*}, k^{*}, v^{*}, w^{*}, s^{*}, t^{*}, \bar{y}^{*}, p^{*}=0\right)$ is a feasible solution of (FD). In addition, if the hypotheses of the weak duality theorem are satisfied for all feasible solutions $(z, \mu, k, v, w, s, t, \bar{y}, p)$ of (FD), then $\left(x^{*}, \mu^{*}, k^{*}, v^{*}, w^{*}, s^{*}, t^{*}, \bar{y}^{*}, p^{*}=0\right)$ is an optimal solution of (FD), and the two objectives have the same optimal values.

Proof Since $x^{*}$ is an optimal solution of (NFP) and $\nabla h_{j}\left(x^{*}\right), j \in J\left(x^{*}\right)$ are linearly independent then, by Theorem 1 , there exist $\left(s^{*}, t^{*}, \bar{y}^{*}\right) \in k\left(x^{*}\right)$ and $\left(x^{*}, \mu^{*}, k^{*}, v^{*}, w^{*}, p^{*}=0\right) \in$ $H_{1}\left(s^{*}, t^{*}, \bar{y}^{*}\right)$ such that $\left(x^{*}, \mu^{*}, k^{*}, v^{*}, w^{*}, p^{*}=0\right)$ is a feasible solution of (FD) and the two objectives have the same values. Optimality of $\left(x^{*}, \mu^{*}, k^{*}, v^{*}, w^{*}, p^{*}=0\right)$ for (FD) thus follows from the weak duality theorem (Theorem 2 ).

Theorem 4 (Strict converse duality) Let $x^{*}$ and $\left(z^{*}, \mu^{*}, k^{*}, v^{*}, w^{*}, s^{*}, t^{*}, \bar{y}^{*}, p^{*}\right)$ be optimal solutions of (NFP) and (FD), respectively, and suppose that $\nabla h_{j}\left(x^{*}\right), j \in J\left(x^{*}\right)$ are linearly independent and there exist $\mathcal{F}, \theta, \phi, b$ and $\rho$ such that

$$
\begin{aligned}
& b\left(x^{*}, z^{*}\right) \phi\left[\sum_{i=1}^{s^{*}} t_{i}\left(f\left(x^{*}, \bar{y}_{i}^{*}\right)+x^{* T} C w^{*}-k^{*}\left(g\left(x^{*}, \bar{y}_{i}^{*}\right)-x^{* T} D v^{*}\right)\right)\right. \\
& -\sum_{i=1}^{s^{*}} t_{i}\left(f\left(z^{*}, \bar{y}_{i}^{*}\right)+z^{* T} C w^{*}-k^{*}\left(g\left(z^{*}, \bar{y}_{i}^{*}\right)-z^{* T} C v^{*}\right)\right) \\
& -\sum_{j=1}^{m} \mu_{j}^{*} h_{j}\left(z^{*}\right)+\frac{1}{2} p^{* T} \nabla^{2} \sum_{i=1}^{s^{*}} t_{i}\left(f\left(z^{*}, \bar{y}_{i}^{*}\right)-k^{*} g\left(z^{*}, \bar{y}_{i}^{*}\right)\right) p^{*} \\
& \left.+\frac{1}{2} p^{* T} \nabla^{2} \sum_{j=1}^{m} \mu_{j}^{*} h_{j}\left(z^{*}\right) p^{*}\right] \leq 0
\end{aligned}
$$




$$
\begin{aligned}
\Rightarrow \quad \mathcal{F}\left(x^{*}, z^{*}, \sum_{i=1}^{s^{*}} t_{i}\left(\nabla f\left(z^{*}, \bar{y}_{i}^{*}\right)+\nabla^{2} f\left(z^{*}, \bar{y}_{i}^{*}\right) p^{*}\right.\right. \\
\left.+C w^{*}-k^{*}\left(\nabla g\left(z^{*}, \bar{y}_{i}^{*}\right)+\nabla^{2} g\left(z^{*}, \bar{y}_{i}^{*}\right) p^{*}-D v^{*}\right)\right) \\
\left.\quad+\sum_{j=1}^{m}\left(\nabla \mu_{j} h_{j}\left(z^{*}\right)+\nabla^{2} \mu_{j} h_{j}\left(z^{*}\right) p^{*}\right)\right) \\
<-\rho\left\|\theta\left(x^{*}, z^{*}\right)\right\|^{2} .
\end{aligned}
$$

Further assume that

$$
\begin{aligned}
& a<0 \Rightarrow \phi(a) \leq 0, \\
& b\left(x^{*}, z^{*}\right)>0, \\
& \rho \geq 0 .
\end{aligned}
$$

Then $z^{*}=x^{*}$, that is, $z^{*}$ is an optimal solution of (NFP).

Proof We shall assume that $x^{*} \neq z^{*}$ and reach a contradiction. Since $x^{*}$ and $\left(z^{*}, \mu^{*}, k^{*}, s^{*}\right.$, $\left.t^{*}, \bar{y}^{*}, v^{*}, w^{*}, p^{*}=0\right)$ are optimal solutions of (NFP) and (FD), respectively, and $\nabla h_{j}\left(x^{*}\right)$, $j \in J\left(x^{*}\right)$ are linearly independent, therefore, from the strong duality theorem (Theorem 3 ), it follows that

$$
\frac{f\left(x^{*}, \bar{y}_{i}^{*}\right)+\left(x^{* T} C x^{*}\right)^{\frac{1}{2}}}{g\left(x^{*}, \bar{y}_{i}^{*}\right)-\left(x^{* T} D x^{*}\right)^{\frac{1}{2}}}=k^{*} .
$$

Thus, we have

$$
\left(f\left(x^{*}, \bar{y}_{i}^{*}\right)+\left(x^{* T} C x^{*}\right)^{\frac{1}{2}}-k^{*}\left(g\left(x^{*}, \bar{y}_{i}^{*}\right)-\left(x^{* T} D x^{*}\right)^{\frac{1}{2}}\right)\right) \leq 0
$$

for all $\bar{y}_{i}^{*} \in Y\left(x^{*}\right), i=1,2, \ldots, s^{*}$.

Now, proceeding as in Theorem 3, we get

$$
\begin{aligned}
& \sum_{i=1}^{s^{*}} t_{i}\left(f\left(x^{*}, \bar{y}_{i}^{*}\right)+x^{* T} C w^{*}-k^{*}\left(g\left(x^{*}, \bar{y}_{i}^{*}\right)-x^{* T} D v^{*}\right)\right) \\
& \quad-\sum_{i=1}^{s^{*}} t_{i}\left(f\left(z^{*}, \bar{y}_{i}^{*}\right)+z^{* T} C w^{*}-k^{*}\left(g\left(z^{*}, \bar{y}_{i}^{*}\right)-z^{* T} D v^{*}\right)\right) \\
& \quad-\sum_{j=1}^{m} \mu_{j}^{*} h_{j}\left(z^{*}\right)+\frac{1}{2} p^{* T} \nabla^{2} \sum_{i=1}^{s^{*}} t_{i}\left(f\left(z^{*}, \bar{y}_{i}^{*}\right)-k^{*} g\left(z^{*}, \bar{y}_{i}^{*}\right)\right) p^{*} \\
& +\frac{1}{2} p^{* T} \nabla^{2} \sum_{j=1}^{m} \mu_{j}^{*} h_{j}\left(z^{*}\right) p^{*} \leq 0 .
\end{aligned}
$$


Using (21) and (22), it follows from (26) that

$$
\begin{aligned}
& b\left(x^{*}, z^{*}\right) \phi\left[\sum_{i=1}^{s^{*}} t_{i}\left(f\left(x^{*}, \bar{y}_{i}^{*}\right)+x^{* T} C w^{*}-k^{*}\left(g\left(x^{*}, \bar{y}_{i}^{*}\right)-x^{* T} D v^{*}\right)\right)\right. \\
& -\sum_{i=1}^{s^{*}} t_{i}\left(f\left(z^{*}, \bar{y}_{i}^{*}\right)+z^{* T} C w^{*}-k^{*}\left(g\left(z^{*}, \bar{y}_{i}^{*}\right)-z^{* T} C v^{*}\right)\right) \\
& -\sum_{j=1}^{m} \mu_{j}^{*} h_{j}\left(z^{*}\right)+\frac{1}{2} p^{* T} \nabla^{2} \sum_{i=1}^{s^{*}} t_{i}\left(f\left(z^{*}, \bar{y}_{i}^{*}\right)-k^{*} g\left(z^{*}, \bar{y}_{i}^{*}\right)\right) p^{*} \\
& \left.+\frac{1}{2} p^{* T} \nabla^{2} \sum_{j=1}^{m} \mu_{j}^{*} h_{j}\left(z^{*}\right) p^{*}\right] \leq 0,
\end{aligned}
$$

which along with (20) and (23) implies

$$
\begin{aligned}
& \mathcal{F}\left(x^{*}, z^{*} ; \sum_{i=1}^{s^{*}} t_{i}\left(\nabla f\left(z^{*}, \bar{y}_{i}^{*}\right)+\nabla^{2} f\left(z^{*}, \bar{y}_{i}^{*}\right) p^{*}\right.\right. \\
& \left.+C w^{*}-k^{*}\left(\nabla g\left(z^{*}, \bar{y}_{i}^{*}\right)+\nabla^{2} g\left(z^{*}, \bar{y}_{i}^{*}\right) p^{*}-D v^{*}\right)\right) \\
& \left.\quad+\sum_{j=1}^{m}\left(\nabla \mu_{j}^{*} h_{j}\left(z^{*}\right)+\nabla^{2} \mu_{j}^{*} h_{j}\left(z^{*}\right) p^{*}\right)\right)<0,
\end{aligned}
$$

which contradicts $(7)$ since $\mathcal{F}\left(x^{*}, z^{*} ; 0\right)=0$.

\section{Conclusion and further development}

In this paper, weak, strong and strict converse duality theorems have been discussed for nondifferentiable minimax fractional programming problems in the framework of generalized convexity type assumptions. This paper has generalized the results of Ahmad [13].

The question arises as to whether the results developed in this paper hold for the following complex nondifferentiable minimax fractional problem:

$$
\begin{array}{ll}
\text { Minimize } & \psi(\xi)=\sup _{v \in W} \frac{\operatorname{Re}\left[f(\xi, v)+\left(z^{T} C z\right)^{\frac{1}{2}}\right]}{\operatorname{Re}\left[g(\xi, v)-\left(z^{T} D z\right)^{\frac{1}{2}}\right]} \\
\text { subject to } & -h(z) \in S, \quad \xi \in C^{2 n},
\end{array}
$$

where $\xi=(z, \bar{z}), v=(w, w)$ for $z \in C^{n}, w \in C^{l}, f(\cdot, \cdot), g(\cdot, \cdot): C^{2 n} \times C^{2 l} \rightarrow C$ are analytic with respect to $W, W$ is a specified compact subset in $C^{2 l}, S$ is a polyhedral cone in $C^{m}$, and $g: C^{2 n} \rightarrow C^{m}$ is analytic. Also, $C, D \in C^{n \times n}$ are positive semidefinite Hermitian matrices.

The author declares that they have no competing interests.

\section{Acknowledgements}

The work is supported by the Deanship of Scientific Research, University of Tabuk, K.S.A. 


\section{References}

1. Barrodale, I: Best rational approximation and strict quasiconvexity. SIAM J. Numer. Anal. 10, 8-12 (1973)

2. Schroeder, RG: Linear programming solutions to ratio games. Oper. Res. 18, 300-305 (1970)

3. Soyster, A, Lev, B, Loof, D: Conservative linear programming with mixed multiple objectives. Omega 5, 193-205 (1977)

4. Bajona-Xandri, C, Martinez-Legaz, JE: Lower subdifferentiability in minimax programming involving type-I functions. J. Comput. Appl. Math. 215, 91-102 (2008)

5. Yadav, SR, Mukherjee, RN: Duality for fractional minimax programming problems. J. Aust. Math. Soc. Ser. B, Appl. Math 31, 484-492 (1990)

6. Chandra, S, Kumar, V: Duality in fractional minimax programming. J. Aust. Math. Soc. A 58, 376-386 (1995)

7. Liu, JC, Wu, CS: On minimax fractional optimality conditions with invexity. J. Math. Anal. Appl. 219, 21-35 (1998)

8. Liu, JC, Wu, CS: On minimax fractional optimality conditions ( $F, \rho)$-invexity. J. Math. Anal. Appl. 219, 36-51 (1998)

9. Ahmad, I: Optimality conditions and duality in fractional minimax programming involving generalized $\rho$-invexity. Int. J. Stat. Manag. Syst. 19, 165-180 (2003)

10. Mangasarian, OL: Second and higher order duality in nonlinear programming. J. Math. Anal. Appl. 51, 607-620 (1975)

11. Mond, B: Second order duality for nonlinear programs. Opsearch 11(2-3), 90-99 (1974)

12. Bector, CR, Chandra, S: Generalized convexity and higher order duality for fractional programming. Opsearch 24(3), 143-154 (1975)

13. Ahmad, I: On second-order duality for minimax fractional programming problems with generalized convexity. Abstr. Appl. Anal. (2011). doi:10.1155/2011/563924

14. Lai, HC, Liu, JC, Tanaka, K: Necessary and sufficient conditions for minimax fractional programming. J. Math. Anal. Appl. 230(2), 311-328 (1999)

10.1186/1029-242X-2013-500

Cite this article as: Khan: Second-order duality for nondifferentiable minimax fractional programming problems with generalized convexity. Journal of Inequalities and Applications 2013, 2013:500

\section{Submit your manuscript to a SpringerOpen ${ }^{\circ}$ journal and benefit from:}

- Convenient online submission

Rigorous peer review

- Immediate publication on acceptance

Open access: articles freely available online

- High visibility within the field

- Retaining the copyright to your article 